\title{
Recurrent Mutations within the Amino-Terminal Region of $\beta$-Catenin Are Probable Key Molecular Driver Events in Sinonasal Hemangiopericytoma
}

Florian Haller, ${ }^{*}$ Matthias Bieg, ${ }^{\dagger}$ Evgeny A. Moskalev, ${ }^{*}$ Sarah Barthelmeß, ${ }^{*}$ Helene Geddert, ${ }^{\ddagger}$ Carsten Boltze ${ }^{\S}$ Nicolle Diessl, Karin Braumandl, ${ }^{*}$ Benedikt Brors, ${ }^{* * \dagger \dagger}$ Heinrich Iro, ${ }^{\ddagger}$ Arndt Hartmann, ${ }^{*}$ Stefan Wiemann, ${ }^{\llbracket \S \S}$ and Abbas Agaimy ${ }^{*}$

\author{
From the Institute of Pathology* and the Department of Otorhinolaryngology, Head and Neck Surgery, ${ }^{\ddagger \ddagger}$ Friedrich Alexander University Erlangen- \\ Nuremberg, Erlangen; the Divisions of Theoretical Bioinformatics, ${ }^{\dagger}$ Applied Bioinformatics, $\|$ and Molecular Genome Analysis ${ }^{\S \S}$ and the Genomics and \\ Proteomics Core Facility, " German Cancer Research Center, Heidelberg; the Institute of Pathology, ${ }^{\ddagger}$ St Vincent’s Hospital, Karlsruhe; the Institute of \\ Pathology, ${ }^{\S}$ SRH-Klinikum, Gera; the National Center for Tumor Diseases (NCT), ** Heidelberg; and the German Consortium for Translational Cancer \\ Research, ${ }^{\dagger \dagger}$ Heidelberg, Germany
}

Accepted for publication October 22, 2014

Address correspondence to Florian Haller, M.D., Ph.D., Institute of Pathology, Friedrich-Alexander University Erlangen-Nuremberg, Krankenhausstr. 8-10, D-91054 Erlangen, Germany. E-mail: florian.haller@uk-erlangen.de.

\begin{abstract}
Sinonasal hemangiopericytoma (SN-HPC) is an uncommon, site-specific, low-grade mesenchymal neoplasm of probable perivascular myoid cell origin. In contrast to solitary fibrous tumors of soft tissue and sinonasal tract origin, SN-HPCs were recently shown to lack recurrent NAB2-STAT6 fusion variants. Other molecular alterations known to occur in some of soft tissue perivascular myoid cell neoplasms were also absent in SN-HPC; thus, the molecular pathogenesis of SN-HPCs remained unknown. Guided by whole-genome sequencing combined with RNA sequencing of an index case, we analyzed a total of six SN-HPCs for mutations within the amino-terminal region of the gene CTNNB1 (cadherin-associated protein), $\beta 1,88 \mathrm{kDa}$, encoding $\beta$-catenin. All six cases showed missense mutations, with amino acid substitutions clustering at positions 33 to 45 , corresponding to the recognition site of the $\beta$-catenin destruction complex. Similar CTNNB1 mutations have been described in a variety of epithelial and mesenchymal neoplasms. These mutations prevent $\beta$-catenin phosphorylation and proteasomal degradation but promote its nuclear accumulation and subsequent increased transcription of Winglessrelated integration site target genes. Consistent with these molecular findings, $\beta$-catenin IHC showed consistent diffuse and strong nuclear staining of the tumor cells in all six SN-HPCs. Our results highlight, for the first time, CTNNB1 mutations as the likely initiating molecular events driving SN-HPC tumorigenesis, which places SN-HPC among the growing family of $\beta$-catenin-driven mesenchymal neoplasms. (Am J Pathol 2015, 185: 563-571; http://dx.doi.org/10.1016/j.ajpath.2014.10.019)
\end{abstract}

Sinonasal hemangiopericytoma (SN-HPC) is a peculiar mesenchymal neoplasm of perivascular myoid cell origin occurring in the nasal cavity and the paranasal sinuses. ${ }^{1,2}$ It accounts for $<0.5 \%$ of all sinonasal neoplasms and is characterized by frequent local recurrence, whereas metastases are exceedingly rare. ${ }^{1,3}$ On the basis of its distinctive morphological features and immunophenotype, SN-HPC has been separated from solitary fibrous tumor (SFT), which can rarely occur in the sinonasal tract, and accounts for $<0.1 \%$ of all sinonasal neoplasms. ${ }^{1,2}$ Recently, recurrent translocations involving the genes NGFI-A binding protein 2 (NAB2) and signal transducer and activator of transcription 6 (STAT6) have been identified as the key molecular driver events in pulmonary and soft tissue SFTs, ${ }^{4-6}$ which are accompanied by aberrant nuclear expression of STAT6. ${ }^{7,8}$ In a recent study, we demonstrated the absence of NAB2-STAT6 translocations in SN-HPCs in contrast to their presence in SFTs arising in the sinonasal tract. ${ }^{9}$ The latter harbor the same NAB2-STAT6 translocations as their pulmonary and soft tissue counterparts. ${ }^{8,9}$ Consistent with this observation, SFTs of the sinonasal tract demonstrated a strong nuclear

Supported by the Institute of Pathology, University of ErlangenNuremberg, Erlangen, Germany, institutional funding and the German Cancer Research Center-Heidelberg Center for Personalized Oncology institutional funding.

Disclosures: None declared. 
expression of STAT6, whereas SN-HPCs did not. ${ }^{9}$ In the current study, we used whole-genome sequencing combined with RNA sequencing (RNA-seq) in a case of SN-HPC with available fresh-frozen tissue to explore the molecular genetic driver events in SN-HPCs. We observed a missense mutation at serine 33 (c.98C $>$ T; p.S33F) in the gene CTNNB1 (cadherin-associated protein), $\beta 1,88 \mathrm{kDa}$, encoding for $\beta$-catenin, a key molecule of the Wingless-related integration site (Wnt) signaling pathway. ${ }^{10}$ Missense mutations in the amino-terminal region of $\beta$-catenin affecting codons 32 to 45 have been described in a variety of epithelial and mesenchymal neoplasms, including colorectal carcinoma, hepatocellular carcinoma, and desmoid-type fibromatosis, and their function in maintaining aberrant activation of the Wnt signaling pathway has been well demonstrated. ${ }^{10-12}$ By using direct Sanger sequencing of CTNNB1 exon 3 in our recently described cohort of six SN-HPCs, ${ }^{9}$ we could demonstrate that missense mutations affecting the aminoterminal region of CTNNB1 are frequent events observed in all six SN-HPCs (100\%). Mutations affecting this region have been shown to prevent degradation by the $\beta$-catenin destruction complex, thus disrupting an important regulation mechanism resulting in nuclear accumulation of $\beta$-catenin. ${ }^{11,12}$ Consistent with this, all six SN-HPCs displayed a diffuse and strong nuclear immunoreactivity for $\beta$-catenin. In summary, this study describes, for the first time, recurrent $C T N N B 1$ mutations as the probable key molecular driver events in SN-HPCs, and provides further genetic evidence that SN-HPC is a site-specific distinctive tumor entity that can be clearly separated from other mesenchymal neoplasms of the sinonasal tract, including SFTs.

\section{Materials and Methods}

\section{Patient Cohort}

The demographic, clinicopathological, and immunohistochemical (IHC) features of the current series of six SNHPCs (Table 1) have been described recently in detail. ${ }^{9}$ All cases have been reclassified by an experienced head and neck and soft tissue pathologist (A.A.), according to the current World Health Organization classification of head and neck tumors. ${ }^{2}$ The patient's age ranged from 49 to 82 years (mean, 72 years) with equal sex distribution. The tumors were confined to the sinonasal tract, and none of the patients developed local recurrence or distant metastasis after resection of the primary tumor. The mean tumor size was $4.4 \mathrm{~cm}$ (range, 1 to $5.5 \mathrm{~cm}$ ). All six cases displayed consistent morphological features of SN-HPC (Figure 1, A-D) with ovoid to spindle-shaped tumor cells with bluntended or rounded uniform nuclei and pale-eosinophilic cytoplasm. The tumor cells were arranged in short fascicles in some areas, whereas other regions displayed a solid growth with occasional glomoid cellular features. Characteristic hyalinized vascular spaces with staghorn-like configuration and focal prominent peritheliomatous fibrosis were present in all cases. By IHC, all six tumors displayed consistent expression of smooth muscle actin. CD34 immunostaining was limited and patchy in four of the cases, and absent in the other three cases. Four cases were variably positive for CD99, and all six cases were negative for bcl2. None of the tumors harbored a NAB2-STAT6 translocation, and all six tumors were negative for STAT6 immunostaining. ${ }^{9}$ From case 1, fresh-frozen tissue was available for whole-genome sequencing and RNA-seq, whereas representative formalin-fixed, paraffin-embedded (FFPE) tissue blocks were used in the other five cases. This study has been approved by the local ethics committee of the University Hospital Erlangen (Erlangen, Germany) (number 217_12 B, 19.09.2012). Signed informed consent was obtained from all participating patients in this study.

\section{Whole-Genome Sequencing}

DNA was isolated from the tumor tissue of an SN-HPC (Case 1) with available fresh-frozen tissue using the DNeasy Blood and Tissue Kit (Qiagen, Hilden, Germany). Quality of DNA was analyzed with an Agilent 2200 Tapestation (Agilent, Waldbronn, Germany). A total load of $1 \mu \mathrm{g}$ DNA was fragmented (Covaris E220; Covaris, Woburn, MA) and size was selected to

Table 1 Patient and Tumor Characteristics of Six SN-HPCs with CTNNB1 Mutations

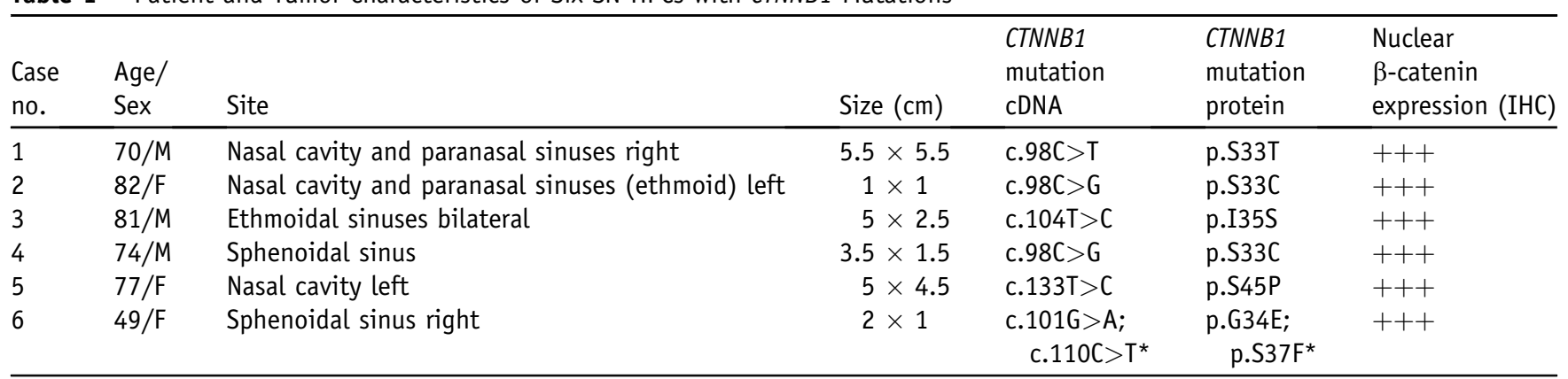

Note that the cases have been published previously. ${ }^{9}$

*This mutation was homozygous.

F, female; M, male; IHC, immunohistochemistry; +++ , strong and diffuse expression. 

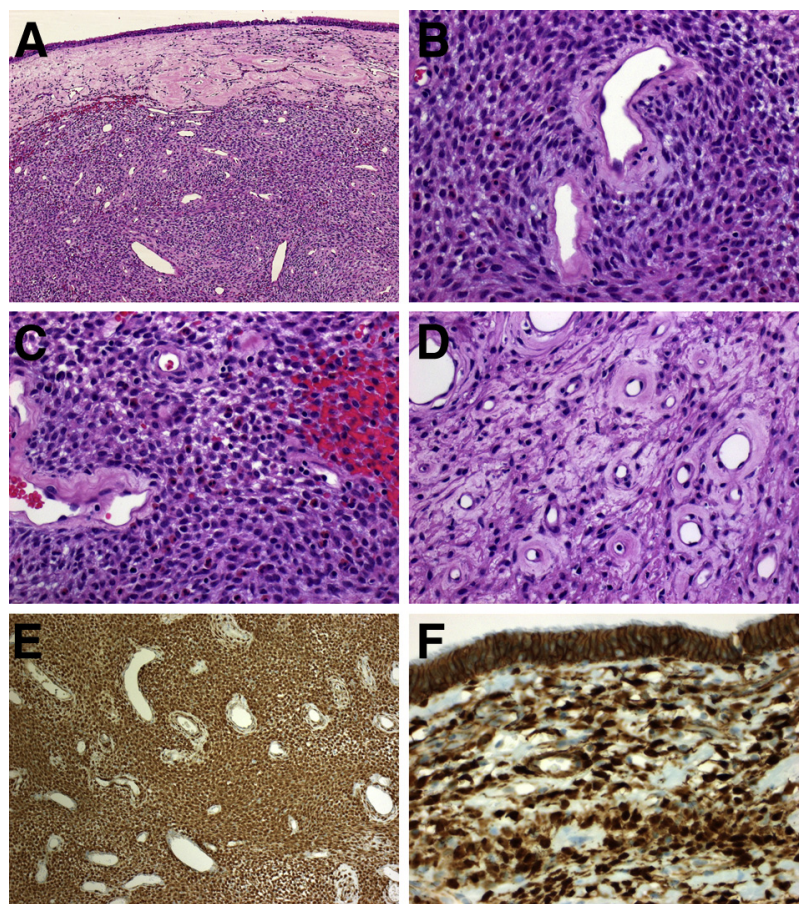

Figure 1 Histomorphological features and $\beta$-catenin immunohistochemistry of sinonasal hemangiopericytoma (SN-HPC). A: SN-HPC covered by intact respiratory epithelium or squamous cell metaplasia. B: The tumor cells are ovoid to spindle shaped with blunt-ended uniform nuclei, sometimes arranged in a whorled pattern around the vessels. C and D: Extravasated erythrocytes and inflammatory infiltrates of mast cells and eosinophilic granulocytes are frequent findings; note the prominent glomoid cellular features. Hyalinized vascular spaces are characteristic for SN-HPC, with staghorn-like configuration (A) and focal prominent peritheliomatous fibrosis (D). E: Immunoreactivity for $\beta$-catenin is homogeneous diffuse and strong in all six cases. F: Note the strong nuclear staining in the tumor cells, indicating nuclear accumulation of the mutated protein, in contrast to physiological membranous staining in the respiratory epithelium. Original magnifications: $\times 100$ [hematoxylin and eosin (H\&E); A]; $\times 400$ (H\&E; B-D); $\times 100$ ( $\beta$-catenin; E); $\times 400$ ( $\beta$-catenin; F).

300 bp using an E-Gel system (Life Technologies, Darmstadt, Germany) in the course of library preparation with the NEBNext DNA Library Prep Master Mix Set and the NEBNext Multiplex Oligos (NEB, Frankfurt, Germany) for Illumina (Illumina, Inc., San Diego, CA), using the supplier's recommendations, and with 10 PCR cycles. Paired-end sequencing $(2 \times 100 \mathrm{bp})$ was performed using three lanes of a HiSeq2000 instrument (Illumina, Inc.). In total, 1,301,994,406 reads were collected to obtain a mean coverage of $\times 33$.

Whole-genome sequencing reads were aligned against the $1-\mathrm{KG}$ phase 2 reference genome (hs37d5; 1000 Genomes Project, http://www.1000genomes.org/data, last accessed October 13, 2014) using an in-house pipeline, as described previously. ${ }^{13}$ The workflow uses bwa version 0.6.2-r126-tpx ${ }^{14}$ and allows for soft-clipped reads requiring a PHRED-based base quality score of at least 20. Each sequencing lane was aligned separately and subsequently merged using samtools version 0.1.17. ${ }^{15}$ PCR duplicates were marked using PICARD tools version 1.95 ( $h$ ttp://picard.sourceforge.net, last accessed October 13, 2014).
For single-nucleotide variation calling, we performed the method, as described recently, combining samtools mpileup version 0.1 .17 and bcftools. ${ }^{13,15}$ Gene annotation and functional impact assignment within coding portions of genes was done by ANNOVAR ${ }^{16}$ using the GENCODE gene model version $17 .{ }^{17}$ Single-nucleotide variations were compared against dbSNP version 135 (http://www.ncbi.nlm. nih.gov/SNP, last accessed October 13, 2014), Online Mendelian Inheritance of Man (http://www.omim.org, last accessed October 13, 2014), and COSMIC (Catalogue of Somatic Mutations in Cancer) version 66 (http://cancer. sanger.ac.uk/cancergenome/projects/cosmic, last accessed October 13, 2014). ${ }^{18}$ In addition, breakpoints of translocations were identified using the CREST (clipping reveals structure) algorithm, which makes use of soft-clipped reads to discover genomic locations with a high proportion of partially mapped reads. ${ }^{19}$

\section{RNA-Seq}

RNA was isolated from the same tumor (Case 1) using the RNeasy Plus Mini Kit (Qiagen). Quality of RNA was assessed using an Agilent Bioanalyzer 2100 (Agilent). For RNA-seq, $1 \mu \mathrm{g}$ of total RNA was fragmented to a median length of $150 \mathrm{bp}$ (Covaris), and library preparation was done with an Illumina TruSeq RNA Sample Preparation Kit version 2, following the supplier's instructions (Illumina, Inc.). Paired-end sequencing $(2 \times 100 \mathrm{bp})$ was performed, and $171,763,248$ reads were collected using a HiSeq2000 instrument (Illumina, Inc.).

Paired-end RNA-seq reads of single lanes were aligned against the 1-KG phase 2 reference genome (hs37d5; 1000 Genomes project, http://www.1000genomes.org/data, last accessed July 21, 2014) separately using STAR version 2.3.0e. ${ }^{20}$ For improving the accuracy of the alignment, we supplied a splice junction database that is based on the GENCODE gene model version $17 .^{17}$ After alignment, single lanes were merged using samtools version $0.1 .17^{15}$ and marked for PCR duplicates using PICARD tools version 1.95 (http://picard.sourceforge.net, last accessed July 20, 2014). Reads per kilobase of transcript per million mapped reads values per gene were calculated using custom perl scripts on the basis of the following formula:

$$
\operatorname{rpkm}=[(\operatorname{reads} / \text { exonSum }) / 1000] /\left(\operatorname{readsTotal} / 10^{6}\right)
$$

Here, reads is the number of mapped non-duplicatemarked reads in the gene, exonSum is the total length of all exons among the gene, and readsTotal is the total number of mapped non-duplicate-marked reads among all exons. For defining exonic and intragenic regions, we used the gene model of The Reference Sequence Database. ${ }^{21}$

Gene-fusion detection on RNA-seq data was performed using DEFUSE version 0.6.1. ${ }^{22}$ Because DEFUSE aligns the reads independently, we supplied the hg19 reference genome and the ENSEMBL version 69 gene and transcript model. $^{23}$ 


\section{Mutation Analysis of CTNNB1 Exon 3 by Sanger} Sequencing

DNA was isolated from FFPE tumor samples using the QIAamp DNA FFPE Tissue Kit (Qiagen). PCR amplification of $C T N N B 1$ exon 3 was performed in $50-\mu \mathrm{L}$ reactions that contained approximately $200 \mathrm{ng}$ DNA, $1.5 \mathrm{mmol} / \mathrm{L} \mathrm{MgCl}_{2}$, $200 \mathrm{mmol} / \mathrm{L}$ dNTP, $500 \mathrm{nmol} / \mathrm{L}$ primers, and $1.25 \mathrm{U}$ Taq DNA polymerase (Genaxxon Bioscience GmbH, Ulm, Germany). Amplification was started by an initial denaturation step at $94^{\circ} \mathrm{C}$ for 3 minutes. The first amplification cycle was denaturation at $94^{\circ} \mathrm{C}$ for 30 seconds, annealing at $65^{\circ} \mathrm{C}$ for 30 seconds, and elongation at $72^{\circ} \mathrm{C}$ for 40 seconds. This procedure was continued for 10 cycles, reducing the annealing temperature by $1^{\circ} \mathrm{C}$ each cycle, followed by 45 cycles of denaturation at $94^{\circ} \mathrm{C}$ for 30 seconds, annealing at $55^{\circ} \mathrm{C}$ for 1 minute, and elongation at $72^{\circ} \mathrm{C}$ for 40 seconds. The sequences of the PCR primers were as follows: 5'-TTTGATGGAGTTGGACATGG-3' (forward) and 5'-CCAGCTACTTGTTCTTGAGTGAA-3' (reverse). Approximately $5 \mu \mathrm{L}$ of each reaction was examined on $2 \%$ agarose gels. Amplicons were sequenced with Sanger chemistry by using the following sequencing primers: 5'-GCCATGGAACCAGACAGAAA-3' (forward) and 5'-AAAATCCCTGTTCCCACTCA-3' (reverse).

\section{$\beta$-Catenin IHC}

The IHC was performed on freshly cut sections ( $3 \mu \mathrm{m}$ thick) of representative FFPE tumor blocks on a fully automated slide preparation and staining system (Benchmark XT System; Ventana Medical Systems Inc., Tuscon, AZ). After pretreatment with buffer $\mathrm{CC} 1$ for $95^{\circ} \mathrm{C}$ for 64 minutes, a mouse monoclonal antibody directed against amino acids 571 to 781 of $\beta$-catenin (clone $14 / \beta$-catenin, catalog number 610154; BD Biosciences, Heidelberg, Germany) was used in a $1: 50$ dilution with incubation at $37^{\circ} \mathrm{C}$ for 32 minutes. Tissue from desmoid-type fibromatosis and scar tissue were used as external positive and negative controls, respectively. In the SN-HPCs, only nuclear staining was considered, whereas physiological strong membranous staining of respiratory epithelium was available as internal positive control in all cases (Figure 1). In addition, IHC staining with antibodies directed against CD34, $\alpha$ smooth muscle actin, bcl2, CD99, and STAT6 has been performed on all cases, as reported previously. ${ }^{9}$

\section{Results}

Deciphering Key Molecular Driver Events in a Case of SN-HPC by Whole-Genome Sequencing and RNA-Seq

The integrative analysis of whole-genome sequencing and RNA-seq data from the index case (Case 1) revealed the absence of a fusion gene that was represented on both genomic DNA and RNA levels. Alignment of the genomic sequence of the tumor DNA to the reference genome and
Table 2 List of Nonsynonymous Somatic Mutations in ProteinCoding Genes Determined by Whole-Genome Sequencing Compared to RNA Abundance Determined by RNA-Sequencing in a SN-HPC (Case 1)

\begin{tabular}{lllr}
\hline Gene & cDNA & Protein & RPKM \\
\hline CTNNB1 & c.C98T & p.S33F & 541.1 \\
NPEPPS & c.T1058G & p.F353C & 31.9 \\
GIGYF2 & c.C83T & p.T28M & 14.59 \\
ESRRA & c.G1055C & p.R352P & 13.94 \\
LPXN & c.G283A & p.E95K & 13.55 \\
DENND1A & c.G2407C & p.A803P & 11.68 \\
ITGA9 & c.G359A & p.R120H & 4.11 \\
POU2F2 & c.G1329T & p.L443F & 0.83 \\
CHIT1 & c.C1049G & p.A350G & 0.01 \\
CHIT1 & c.G1046A & p.W349X & 0.01 \\
DSPP & c.G3103A & p.D1035N & 0 \\
RHAG & c.C527T & p.A176V & 0 \\
\hline
\end{tabular}

The RPKM value is proportional to the RNA abundance of a gene. RPKM, reads per kilobase of transcript per million mapped reads.

application of filters, as described in Materials and Methods, revealed 12 nonsynonymous somatic mutations within 11 protein-coding genes, including a missense mutation in exon 3 of the CTNNB1 gene (c.98C $>$ T; p.S33F) (Table 2). The CTNNB1 mutation was present on both the genomic DNA and the RNA levels, and this specific missense mutation has been reported in different types of epithelial and mesenchymal neoplasms before (mutation ID COSM5669). The integrative analysis of genes with nonsynonymous somatic mutations in comparison to the RNAseq expression data revealed a high RNA abundance only for CTNNB1, whereas the other genes were only rarely represented on the RNA level (Table 2).

According to the major role of $\beta$-catenin as a central effector molecule of the Wnt pathway and its important function as transcriptional coactivator in the T-cell factor (TCF)/ $\beta$-catenin transcriptional complex, we compared the RNA abundance of selected genes with known function in Wnt signaling and $\mathrm{TCF} / \beta$-catenin target gene expression (Table 3 ). In detail, we evaluated $51 \mathrm{Wnt}$ signaling components with either agonistic or antagonistic effect on the pathway, the 5 components of the $\beta$-catenin destruction complex, 11 components and cofactors of the TCF/ $\beta$-catenin transcriptional complex, and 5 wellknown TCF/ $\beta$-catenin target genes. Interestingly, the highest RNA abundance with reads per kilobase of transcript per million mapped reads values $>150$ was observed for CTNNB1 itself, for three TCF/ $\beta$-catenin target genes (the transcription factors FOS, JUN, and TWISTI) and for four components of the Wnt pathway with antagonistic function (APCDD1, NKD1, NKD2, and WIF1). In contrast, most Wnt agonists displayed low RNA abundance. Collectively, these RNA expression data further support the functional effect of the $\beta$-catenin mutation in the index case, demonstrating upregulation of $\mathrm{TCF} / \beta$-catenin transcriptional target genes in the absence of agonistic Wnt signaling components upstream of the mutated $\beta$-catenin. 
Table 3 RNA-Sequencing Data for Selected Genes Involved in Wnt Signaling and TCF/ $\beta$-Catenin Target Gene Expression

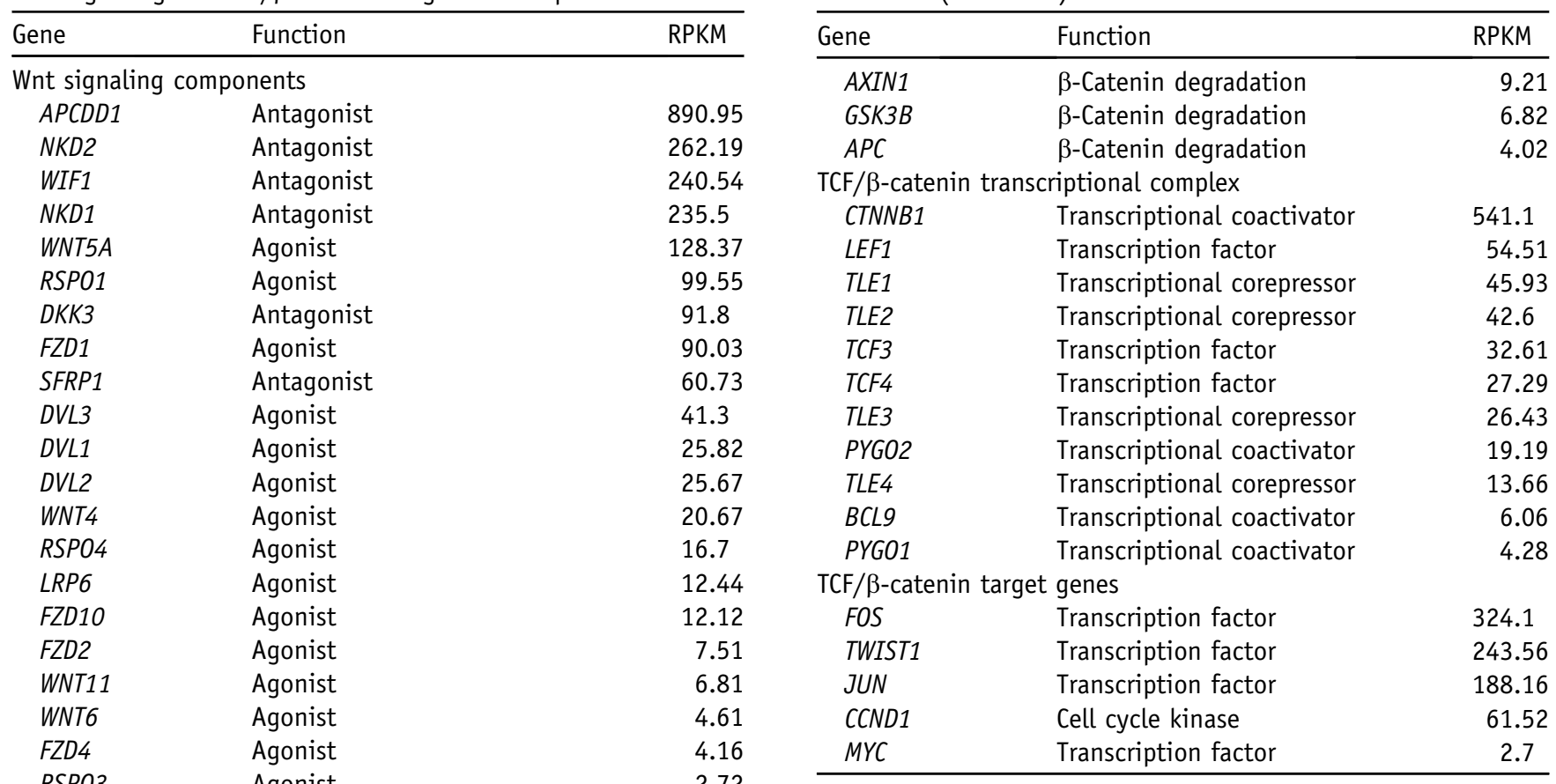

Agonist 2.72

FZD9 Agonist 2.33

$\begin{array}{lll}L R P 5 & \text { Agonist } & 2.18\end{array}$

$\begin{array}{lll}\text { WNT5B } & \text { Agonist } & 1.77\end{array}$

$\begin{array}{lll}\text { WNT3 } & \text { Agonist } & 1.34\end{array}$

$\begin{array}{lll}\text { WNT2B } & \text { Agonist } & 1.32\end{array}$

FZD6 Agonist $\quad 0.66$

FZD3 Agonist 0.61

$\begin{array}{lll}\text { SFRP4 } & \text { Antagonist } & 0.58\end{array}$

WNT10A Agonist 0.38

FZD8 Agonist $\quad 0.26$

$\begin{array}{lll}\text { WNT7B Agonist } & 0.25\end{array}$

DKK4 Antagonist $\quad 0.24$

FZD7 Agonist 0.19

DKK2 Antagonist 0.18

WNT9A Agonist 0.14

FZD5 Agonist 0.13

DKK1 Antagonist $\quad 0.13$

$\begin{array}{lll}\text { WNT1OB } & \text { Agonist } & 0.1\end{array}$

WNT3A Agonist 0.08

$\begin{array}{lll}\text { SFRP2 } & \text { Antagonist } & 0.08\end{array}$

$\begin{array}{lll}\text { WNT8B } & \text { Agonist } & 0.06\end{array}$

$\begin{array}{lll}\text { WNT16 Agonist } & 0.05\end{array}$

$\begin{array}{lll}\text { WNT9B } & \text { Agonist } & 0.04\end{array}$

$\begin{array}{lll}\text { RSPO2 Agonist } & 0.03\end{array}$

$\begin{array}{ll}\text { WNT1 Agonist } & 0.02\end{array}$

$\begin{array}{lll}\text { WNT8A } & \text { Agonist } & 0.01\end{array}$

$\begin{array}{lll}\text { WNT2 } & \text { Agonist } & 0.01\end{array}$

WNT7A Agonist

NDP Agonist

SFRP5 Antagonist

$\beta$-Catenin destruction complex

AXIN2 $\quad \beta$-Catenin degradation $\quad 137.17$

CSNK1A1 $\quad \beta$-Catenin degradation

BTRC $\quad \beta$-Catenin degradation
Table 3 (continued)

The RPKM value is proportional to the RNA abundance of a gene.

RPKM, reads per kilobase of transcript per million mapped reads; TCF, T-cell factor; Wnt, Wingless-related integration site.

\section{Frequent CTNNB1 Mutations in a Cohort of SN-HPCS}

Collectively, the findings in the index case encouraged us to analyze five additional SN-HPCs for mutations in CTNNB1 by Sanger sequencing. The missense mutation affecting serine 33 (p.S33F) found in the index case (Case 1) by whole-genome sequencing was confirmed (Figure 2A). Strikingly, all additional five SN-HPCs harbored similar missense mutations in the amino-terminal region of CTNNB1 (Figure 2, B-F, and Table 1). Two cases also had amino acid substitutions at serine 33 (p.S33C), one case had a substitution at isoleucine 35 (p.I35S), one case had a substitution at serine 45 (p.S45P), and one case had two mutations with substitution of glycine 34 (p.G34E) combined with a homozygous substitution of serine 37 (p.S37F). According to the well-known mechanism of nuclear accumulation of $\beta$-catenin with mutations in the amino-terminal region, the tumors were analyzed for $\beta$-catenin expression by IHC. Consistent with the molecular findings, all six SN-HPCs demonstrated a homogeneous diffuse and strong nuclear staining for $\beta$-catenin (Figure 1, E and F).

\section{Discussion}

Identification of Recurrent CTNNB1 Mutations in SN-HPC

52.71

10.39

Until now, the molecular pathogenesis of SN-HPCs remained obscure. Namely, the recurrent NAB2-STAT6 fusions described 


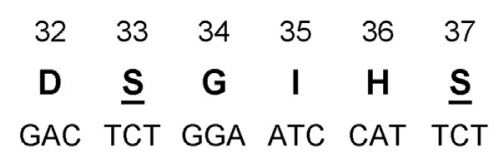

A

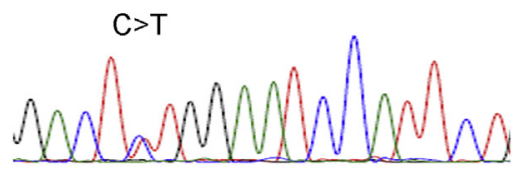

B

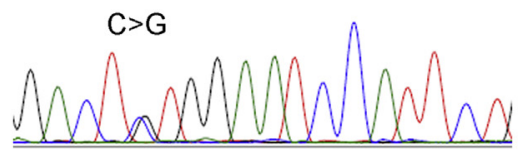

C

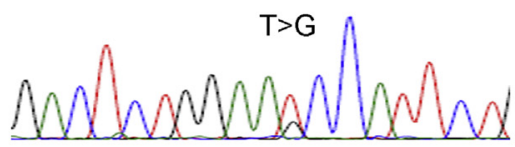

p.S33C

D

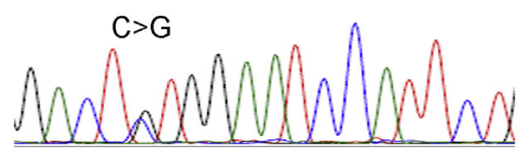

$\mathbf{E}$

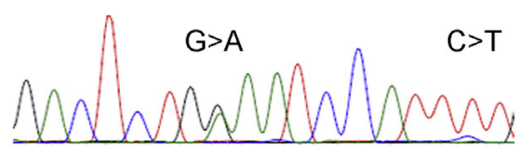

p.G34E

p.S37F*

\section{p.I35S}

p.S33C

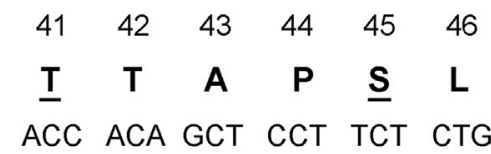

$\mathbf{F}$

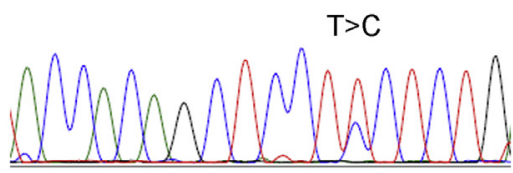

\section{p.T45P}

Figure 2 CTNNB1 Sanger sequencing results for six sinonasal hemangiopericytomas. A: Case 1 (index case) with p.S33F mutation. B: Case 2 with p.S33C mutation. C: Case 3 with p.I35S mutation. D: Case 4 with p.S33C mutation. E: Case 6 with p.G34E mutation and p.S37F mutation. Note the homozygous state of the p.S37F mutation (asterisk). F: Case 5 with p.T45P mutation. The reference sequences for region 32 to 37 and for region 41 to 46 with the encoded amino acids are shown for comparison, with phosphorylation sites underlined.

recently in most soft tissue tumors included in the family of SFT/HPC could not be detected in any of six SN-HPCs examined in a recent study. ${ }^{9}$ Another recent study failed to demonstrate any rearrangements of NOTCH1-3 genes in five cases of SN-HPCs analyzed. ${ }^{24}$ Accordingly, absence of similar alterations in SN-HPCs suggested a different, albeit yet unknown, molecular pathway involved in these morphologically distinctive tumors confined to the sinonasal tract.

Herein, we present, for the first time, strong evidence that recurring missense mutations in exon 3 of the CTNNB1 gene, leading to substitutions of amino acids in the amino-terminal region of $\beta$-catenin, are the probable key molecular driver event in SN-HPCs. These mutations were detected in all six SN-HPCs examined in the current study and were accompanied by consistent nuclear accumulation of $\beta$-catenin, thus providing strong evidence that the reported CTNNB1 mutations are correlated to an oncogenic functional status of $\beta$-catenin in a manner reminiscent of desmoid-type fibromatosis and other $\beta$-catenin-driven neoplasms. ${ }^{11,25,26}$

\section{Regulation and Function of $\beta$-Catenin in the Wnt Signaling Pathway}

$\beta$-Catenin is a key regulatory molecule in the Wnt pathway, an important signaling network controlling embryonic development and adult tissue homeostasis with effects on cell proliferation and differentiation. ${ }^{10}$ In the absence of activating Wnt signals, cytosolic $\beta$-catenin is constantly degraded and its levels are low. After Wnt activation, $\beta$-catenin is stabilized through phosphorylation and translocates to the nucleus, where it forms a transcriptionally active complex with members of the TCF/LEF family of transcription factors and coactivates the expression of prosurvival and proliferative genes. ${ }^{10,27}$ According to its fundamental role in connecting the transcriptional response to on and off states of Wnt signaling pathway, activation of $\beta$-catenin is tightly regulated, but make it susceptible to oncogenic mutations observed in a variety of epithelial and mesenchymal neoplasms. ${ }^{11}$ Specifically, mutations affecting the amino-terminal region at positions 32 to 45 abrogate the phosphorylation-dependent degradation through a multiprotein assembly called the $\beta$-catenin destruction complex. ${ }^{11,12}$ In a first step, serine 45 is phosphorylated by casein kinase 1. Phosphorylated serine 45 then primes further phosphorylation of threonine 41 and subsequently of serines 37 and 33 by glycogen synthase kinase 3 (GSK3).

Additional components of the destruction complex are the adenomatosis polyposis coli (APC) protein, the scaffolding protein Axin, and the E3-ubiquitin ligase $\beta$-TrCP. ${ }^{11,12}$ Collectively, these proteins form the $\beta$-catenin destruction complex, which directs phosphorylated $\beta$-catenin to proteasomal degradation.

\section{CTNNB1 Mutation in SN-HPC Disrupts the Phosphorylation Motive Necessary for $\beta$-Catenin Degradation}

The mutations found in five of six SN-HPCs in the current study directly affected the phosphorylation sites, serine 33 (three cases), serine 37 (one case), and serine 45 (one case), whereas the sixth case harbored a mutation at position 35 with substitution of isoleucine to serine. The substitutions of serines in the first five cases directly foreclose the phosphorylation of $\beta$-catenin by GSK 3 at serines 33 and 37 and by casein kinase 1 at serine 45 , respectively. ${ }^{11,12}$ In the sixth case, the installation of serine at position 35 disrupts the S/T-X-X-X-pS/T sequence motive necessary for phosphorylation of serine 33 by 
GSK3. ${ }^{11,12}$ In summary, all six SN-HPCs harbored mutations abrogating the phosphorylation and subsequent proteasomal degradation of $\beta$-catenin by the $\beta$-catenin disruption complex, which is consistent with a nuclear accumulation of $\beta$-catenin demonstrated by diffuse and strong nuclear immunoreactivity in all six cases.

\section{Expression Analysis of Wnt Signaling Components and $\beta$-Catenin Transcriptional Targets Supports Functional Relevance of CTNNB1 Mutation in SN-HPC}

The RNA-seq data available for the index case enabled us to compare the RNA abundance of 73 components of Wnt signaling and TCF/ $\beta$-catenin transcriptional response. ${ }^{10,27}$ In detail, $51 \mathrm{Wnt}$ signaling components with agonistic or antagonistic effect on the Wnt pathway, the 6 components of the $\beta$-catenin destruction complex, 11 components and cofactors of the TCF/ $\beta$-catenin transcriptional complex, and $5 \mathrm{TCF} / \beta$ catenin target genes were evaluated for their RNA abundance in relation to their main functional effect on Wnt signaling and $\mathrm{TCF} / \beta$-catenin target gene expression. We observed a high RNA abundance for the TCF/ $\beta$-catenin target genes $F O S$, $J U N$, and TWIST1, which constitute three important transcription factors involved in cell proliferation, differentiation, and transformation. ${ }^{10,27}$ Notably, the four components of the Wnt signaling pathway with highest RNA abundance (APCDD1, NKD2, WIF1, and NKD1) have an antagonistic effect on the pathway, and constitute a physiological negative feedback loop with intent to dampen the activated Wnt signaling. ${ }^{10,27}$ In contrast, Wnt signaling components with agonistic effect had a rather low RNA abundance. Collectively, these RNA expression data indicate transcriptional activation of $\mathrm{TCF} / \beta$-catenin target genes located downstream of the mutated $\beta$-catenin in the absence of agonistic Wnt signaling components upstream of $\beta$-catenin, thus further supporting the functional relevance of the observed $\beta$-catenin mutation and nuclear $\beta$-catenin accumulation.

\section{CTNNB1 Mutations in Other Mesenchymal Neoplasms}

Desmoid-type fibromatosis is the prototype soft tissue neoplasm driven by alterations affecting the Wnt signaling network. ${ }^{25,26}$ Although desmoid-type fibromatosis in patients with familial adenomatous polyposis coli (Gardner syndrome) occurs in the context of $A P C$ germ-line mutations, sporadic cases of desmoid-type fibromatosis frequently harbor mutations affecting serine 45 and threonine 41 of CTNNB1. ${ }^{25,26,28,29}$ These amino acid substitutions abrogate $\beta$-catenin phosphorylation through casein kinase 1 and GSK3 and confer to oncogenic stabilization and nuclear accumulation of $\beta$-catenin, which can be visualized by nuclear immunoreactivity. ${ }^{25,26,28,29}$ Moreover, recurrent mutations in the amino-terminal region of CTNNB1 have also been reported in $75 \%$ of nasopharyngeal (juvenile) angiofibromas, ${ }^{30}$ and recently, in $88 \%$ of palisaded myofibroblastoma of lymph node (synonym: intranodal hemorrhagic spindle cell tumor with amianthoid fibers). ${ }^{31}$ In both entities, CTNNB1 mutations were also accompanied by strong nuclear $\beta$-catenin immunoreactivity. ${ }^{30,31}$ Nasopharyngeal (juvenile) angiofibroma is a rare lesion with striking predominance for young males. It occurs in the posterolateral wall of the nasal cavity and the nasopharynx and is characterized by irregular vessels embedded in a fibrous stroma. ${ }^{32}$ Palisaded myofibroblastoma of lymph node represents a rare benign myofibroblastic neoplasm restricted to lymph nodes, mainly of the inguinal region. ${ }^{33,34}$ Thus, SN-HPC represents the fourth documented example of a $\beta$-catenin-driven mesenchymal neoplasm and the first one of possible/probable perivascular myoid cell origin.

\section{Distinct Types of CTNNB1 Mutations in $\beta$-Catenin-Driven Mesenchymal Neoplasms Correlate with Different Local Recurrence Rates}

Interestingly, CTNNB1 mutations in desmoid-type fibromatosis are mainly restricted to serine 45 and threonine $41,{ }^{25,26,28,29}$ with $<3 \%$ of the cases harboring mutations at positions 33,34 , and $37 .{ }^{25}$ In contrast, CTNNB1 mutations in nasopharyngeal angiofibromas and palisaded myofibroblastoma of lymph node cluster within codons 32 to 37 , whereas no mutations at serine 45 and threonine 41 have been documented. $^{30,31}$ In the current series of six SN-HPCs, we observed a similar pattern comparable to the latter two entities with predominant mutations of amino acids 33 to 35 , and only one case displaying a mutation affecting serine 45 . We cannot explain these interesting differences in the affected amino acid positions, but differential activation of components of Wnt signaling network and the $\beta$-catenin destruction complex in the probable different cells of origin of these distinct tumor entities may contribute to these different mutation signatures. Interestingly, site-specific different mutation frequencies have been reported comparing intra-abdominal (mesenteric) with extra-abdominal (desmoid-type) fibromatosis, ${ }^{29,35}$ and mutations involving serine 45 have been recently introduced as an independent prognostic marker for local recurrence. ${ }^{29}$ Accordingly, different mutation sites within the aminoterminal region of CTNNB1 may contribute to the morphological and biological heterogeneity of the well-characterized $\beta$-catenin-driven mesenchymal neoplasms ranging from fully benign lesions (intranodal palisaded myofibroblastoma) to non-metastasizing tumors of varying intermediate biological features, with low (SN-HPC), intermediate (nasopharyngeal angiofibroma), or high (desmoid fibromatosis) local recurrence rates.

\section{Diagnostic and Prognostic Relevance of CTNNB1 Mutation Analysis and $\beta$-Catenin Immunostaining in SN-HPC}

Well-documented distant metastasis from SN-HPCs is almost nonexistent. Among the initial series with the first description of SN-HPC, none of 23 cases metastasized, with 4 (17\%) local 
recurrences. ${ }^{1}$ In the largest published series of 104 wellcharacterized SN-HPCs, 17 (17\%) of 101 cases with followup developed local recurrence after resection, but no distant metastasis was reported. ${ }^{3}$ In that study, three patients (3\%) died with local disease, including two cases with bone invasion, after one to eight local recurrences. ${ }^{3}$ Regarding the hereby introduced CTNNB1 mutations in SN-HPCs and integrating these tumors among other $\beta$-catenin-driven mesenchymal neoplasms with varying potential for local recurrence but absence of any metastatic potential, the few reported examples of highly aggressive SN-HPCs might have represented rare cases of sinonasal SFTs. ${ }^{36}$

From a differential diagnostic point of view, $\beta$-catenin IHC may emerge as an adjunct diagnostic tool in better defining and recognizing SN-HPCs compared with other mesenchymal neoplasms occurring in the sinonasal tract on the one hand. On the other hand, frequent CTNNB1 mutations and $\beta$-catenin reactivity in SN-HPCs, particularly their collagen-rich (fibrous) variants, should be distinguished from rare cases of extra-abdominal desmoid-type fibromatosis originating within the sinonasal tract. $^{37,38}$ In this regard, the classic histopathological features of the two neoplasms are readily distinguishable if sufficient tissue is obtained. Most important, a strong nuclear $\beta$-catenin staining in limited or crushed biopsy specimen of a HPC-like sinonasal lesion should warrant diagnosis of SN-HPCs.

\section{Conclusion}

In summary, we report herein, for the first time, recurrent CTNNB1 missense mutations associated with diffuse and strong nuclear accumulation of $\beta$-catenin in SN-HPCs, pointing to these findings as the most likely initiating molecular driver event in this uncommon site-specific tumor. The potential targeting of these molecular alterations in cases with unresectable disease remains to be assessed in future studies. SN-HPCs must be distinguished from other $\beta$-catenin-positive lesions, such as desmoid-type fibromatosis, which may rarely originate at this anatomical site.

\section{Acknowledgments}

We thank Eva Gerum, Claudia Störer, and Maxi Ziegler (Institute of Pathology Erlangen) for excellent technical assistance, Ute Ernst and Sabine Schmidt [German Cancer Research Center (DKFZ) Genomics and Proteomics Core Facility] for excellent support, and the DKFZ-Heidelberg Center for Personalized Oncology for expertise and funding.

\section{References}

1. Compagno J, Hyams VJ: Hemangiopericytoma-like intranasal tumors: a clinicopathologic study of 23 cases. Am J Clin Pathol 1976, 66: $672-683$
2. Thompson LDR, Fanburg-Smith JC, Wenig BM: Borderline and low malignant potential tumors of soft tissues. Edited by Barnes L, Eveson JW, Reichart P, Sidransky D. World Health Organization Classification of Tumours: Pathology and Genetics of Head and Neck Tumours. Lyon, IARC Press, 2005, pp 43-45

3. Thompson LD, Miettinen M, Wenig BM: Sinonasal-type hemangiopericytoma: a clinicopathologic and immunophenotypic analysis of 104 cases showing perivascular myoid differentiation. Am J Surg Pathol 2003, 27:737-749

4. Robinson DR, Wu YM, Kalyana-Sundaram S, Cao X, Lonigro RJ Sung YS, Chen CL, Zhang L, Wang R, Su F, Iyer MK, Roychowdhury S, Siddiqui J, Pienta KJ, Kunju LP, Talpaz M, Mosquera JM, Singer S, Schuetze SM, Antonescu CR, Chinnaiyan AM: Identification of recurrent NAB2-STAT6 gene fusions in solitary fibrous tumor by integrative sequencing. Nat Genet 2013, 45:180-185

5. Chmielecki J, Crago AM, Rosenberg M, O’Connor R, Walker SR, Ambrogio L, Auclair D, McKenna A, Heinrich MC, Frank DA Meyerson M: Whole-exome sequencing identifies a recurrent NAB2STAT6 fusion in solitary fibrous tumors. Nat Genet 2013, 45:131-132

6. Mohajeri A, Tayebwa J, Collin A, Nilsson J, Magnusson L, von Steyern FV, Brosjö O, Domanski HA, Larsson O, Sciot R, DebiecRychter M, Hornick JL, Mandahl N, Nord KH, Mertens F: Comprehensive genetic analysis identifies a pathognomonic NAB2/STAT6 fusion gene, nonrandom secondary genomic imbalances, and a characteristic gene expression profile in solitary fibrous tumor. Genes Chromosomes Cancer 2013, 52:873-886

7. Doyle LA, Vivero M, Fletcher CD, Mertens F, Hornick JL: Nuclear expression of STAT6 distinguishes solitary fibrous tumor from histologic mimics. Mod Pathol 2014, 27:390-395

8. Barthelmeß S, Geddert H, Boltze C, Moskalev EA, Bieg M, Sirbu H, Brors B, Wiemann S, Hartmann A, Agaimy A, Haller F: Solitary fibrous tumors/hemangiopericytomas with different variants of the NAB2-STAT6 gene fusion are characterized by specific histomorphology and distinct clinicopathological features. Am J Pathol 2014, 184:1209-1218

9. Agaimy A, Barthelmeß S, Geddert H, Boltze C, Moskalev E, Koch M, Wiemann S, Hartmann A, Haller F: Phenotypical and molecular distinctness of sinonasal hemangiopericytoma compared to solitary fibrous tumor of the sinonasal tract. Histopathology 2014, 65:667-673

10. MacDonald BT, Tamai K, He X: Wnt/beta-catenin signaling: components, mechanisms, and diseases. Dev Cell 2009, 17:9-26

11. Polakis P: The oncogenic activation of beta-catenin. Curr Opin Genet Dev 1999, 9:15-21

12. Stamos JL, Weis WI: The $\beta$-catenin destruction complex. Cold Spring Harb Perspect Biol 2013, 5:a007898

13. Jones DT, Hutter B, Jäger N, Korshunov A, Kool M, Warnatz HJ, et al; International Cancer Genome Consortium PedBrain Tumor Project: Recurrent somatic alterations of FGFR1 and NTRK2 in pilocytic astrocytoma. Nat Genet 2013, 45:927-932

14. Li H, Durbin R: Fast and accurate short read alignment with Burrows-Wheeler transform. Bioinformatics 2009, 25:1754-1760

15. Li H, Handsaker B, Wysoker A, Fennell T, Ruan J, Homer N, Marth G, Abecasis G, Durbin R; 1000 Genome Project Data Processing Subgroup: The Sequence Alignment/Map format and SAMtools. Bioinformatics 2009, 25:2078-2079

16. Wang K, Li M, Hakonarson H: ANNOVAR: functional annotation of genetic variants from high-throughput sequencing data. Nucleic Acids Res 2010, 38:e164

17. Harrow J, Frankish A, Gonzalez JM, Tapanari E, Diekhans M, Kokocinski F, et al: GENCODE: the reference human genome annotation for The ENCODE Project. Genome Res 2012, 22:1760-1774

18. Forbes SA, Bindal N, Bamford S, Cole C, Kok CY, Beare D, Jia M, Shepherd R, Leung K, Menzies A, Teague JW, Campbell PJ, Stratton MR, Futreal PA: COSMIC: mining complete cancer genomes in the Catalogue of Somatic Mutations in Cancer. Nucleic Acids Res 2011, 39:D945-D950

19. Wang J, Mullighan CG, Easton J, Roberts S, Heatley SL, Ma J, Rusch MC, Chen K, Harris CC, Ding L, Holmfeldt L, Payne-Turner D, 
Fan X, Wei L, Zhao D, Obenauer JC, Naeve C, Mardis ER, Wilson RK, Downing JR, Zhang J: CREST maps somatic structural variation in cancer genomes with base-pair resolution. Nat Methods 2011, 8:652-654

20. Dobin A, Davis CA, Schlesinger F, Drenkow J, Zaleski C, Jha S, Batut P, Chaisson M, Gingeras TR: STAR: ultrafast universal RNAseq aligner. Bioinformatics 2013, 29:15-21

21. Pruitt K, Brown G, Tatusova T, Maglott D: The Reference Sequence (RefSeq) Database. 2002 Oct 9 [Updated 2012 Apr 6]. Edited by J McEntyre, J Ostell. The NCBI Handbook [Internet]. Bethesda, National Center for Biotechnology Information (US), 2002, Chapter 18. Available at http://www.ncbi.nlm.nih.gov/books/NBK21091

22. McPherson A, Hormozdiari F, Zayed A, Giuliany R, Ha G, Sun MG, Griffith M, Heravi Moussavi A, Senz J, Melnyk N, Pacheco M, Marra MA, Hirst M, Nielsen TO, Sahinalp SC, Huntsman D, Shah SP: deFuse: an algorithm for gene fusion discovery in tumor RNA-Seq data. PLoS Comput Biol 2011, 7:e1001138

23. Flicek P, Amode MR, Barrell D, Beal K, Billis K, Brent S, et al: Ensembl 2014. Nucleic Acids Res 2014, 42:D749-D755

24. Mosquera JM, Sboner A, Zhang L, Chen CL, Sung YS, Chen HW, Agaram NP, Briskin D, Basha BM, Singer S, Rubin MA, Tuschl T, Antonescu CR: Novel MIR143-NOTCH fusions in benign and malignant glomus tumors. Genes Chromosomes Cancer 2013, 52: $1075-1087$

25. Miyoshi Y, Iwao K, Nawa G, Yoshikawa H, Ochi T, Nakamura Y: Frequent mutations in the beta-catenin gene in desmoid tumors from patients without familial adenomatous polyposis. Oncol Res 1998, 10: 591-594

26. Tejpar S, Nollet F, Li C, Wunder JS, Michils G, dal Cin P, Van Cutsem E, Bapat B, van Roy F, Cassiman JJ, Alman BA: Predominance of betacatenin mutations and beta-catenin dysregulation in sporadic aggressive fibromatosis (desmoid tumor). Oncogene 1999, 18:6615-6620

27. Logan CY, Nusse R: The Wnt signaling pathway in development and disease. Annu Rev Cell Dev Biol 2004, 20:781-810

28. Le Guellec S, Soubeyran I, Rochaix P, Filleron T, Neuville A, Hostein I, Coindre JM: CTNNB1 mutation analysis is a useful tool for the diagnosis of desmoid tumors: a study of 260 desmoid tumors and 191 potential morphologic mimics. Mod Pathol 2012, 25:1551-1558
29. Colombo C, Miceli R, Lazar AJ, Perrone F, Pollock RE, Le Cesne A, Hartgrink HH, Cleton-Jansen AM, Domont J, Bovée JV, Bonvalot S, Lev D, Gronchi A: CTNNB1 45F mutation is a molecular prognosticator of increased postoperative primary desmoid tumor recurrence: an independent, multicenter validation study. Cancer 2013, 119:3696-3702

30. Abraham SC, Montgomery EA, Giardiello FM, Wu TT: Frequent betacatenin mutations in juvenile nasopharyngeal angiofibromas. Am J Pathol 2001, 158:1073-1078

31. Laskin WB, Lasota JP, Fetsch JF, Felisiak-Golabek A, Wang ZF, Miettinen M: Intranodal palisaded myofibroblastoma: another mesenchymal neoplasm with CTNNB1 ( $\beta$-catenin gene) mutations: clinicopathologic, immunohistochemical, and molecular genetic study of 18 cases. Am J Surg Pathol 2014, [Epub ahead of print], http://dx.doi.org/ 10.1097/PAS.0000000000000299

32. Martin H, Ehrlich HE, Abels JC: Juvenile nasopharyngeal angiofibroma. Ann Surg 1948, 127:513-536

33. Suster S, Rosai J: Intranodal hemorrhagic spindle-cell tumor with "amianthoid" fibers: report of six cases of a distinctive mesenchymal neoplasm of the inguinal region that simulates Kaposi's sarcoma. Am J Surg Pathol 1989, 13:347-357

34. Weiss SW, Gnepp DR, Bratthauer GL: Palisaded myofibroblastoma: a benign mesenchymal tumor of lymph node. Am J Surg Pathol 1989, 13:341-346

35. Huss S, Nehles J, Binot E, Wardelmann E, Mittler J, Kleine MA, Künstlinger H, Hartmann W, Hohenberger P, Merkelbach-Bruse S, Buettner R, Schildhaus HU: $\beta$-Catenin (CTNNB1) mutations and clinicopathological features of mesenteric desmoid-type fibromatosis. Histopathology 2013, 62:294-304

36. Watanabe K, Saito A, Suzuki M, Yamanobe S, Suzuki T: True hemangiopericytoma of the nasal cavity. Arch Pathol Lab Med 2001, 125:686-690

37. Gnepp DR, Henley J, Weiss S, Heffner D: Desmoid fibromatosis of the sinonasal tract and nasopharynx: a clinicopathologic study of 25 cases. Cancer 1996, 78:2572-2579

38. Flucke U, Tops BB, van Diest PJ, Slootweg PJ: Desmoid-type fibromatosis of the head and neck region in the paediatric population: a clinicopathological and genetic study of seven cases. Histopathology 2014, 64:769-776 